\section{EA STATION DE FORCE DES CHUTES DU STAVE}

La Compagnie des Forces de l'Ouest canadien fut fondée en 1909 pour fournir la force nécessaire aux besoins in dustriels de Vancouver et du voisinage. En juin rgog, la Compagnie prit la propriété des franchises de la Compagnie de force du lac Stave qui avait obtenu une charte du gouvernement de la province de Colombie britannique, hi donnant le droit de prendre la force à la rivière Stave ct de la distribuer dans tout le district entourant Vancouver ef aussi Vancouver et New-Westminster. La Compagnie de force du lac Stave avait, au cours de plusieurs années antérieures à 1909 , fait des travaux préliminaires considérables sir la rivière Stave, comprenant l'établissement d'une station de jauge, la construction de chemins et de camps et la construclion partielle d'une digue à écluses. Ia Compagnic de puissance de l'Oucst canadien commença la consIruclion d'une station de 50 ooo chevaux-vapeur dès l'hiver 1909-1910. Ia première section de cctte station comprenant 2 unités de ro ooo $\mathrm{Kw}$. de capacité chacune, fut achevée et la force distribuéc à Vancouver le ror janvier rgra.

Pendant les années igrx et rgr2, la Compagnie construisit un système de distribution comprenant environ 550 miles de lignes couvrant pratiquement le district entier de Vanconter.

Pondant celte période une grande quantité de force a été vendue ì des industries nouvelles ou existantes. Ie point de développemen tole la force est aux chutes du Stave, environ à 6 miles au Nord de la jonction des rivières Stave et Frather, et à 35 miles à l'Est de Vancouver.

Les montagnes fournissant la nappe d'eau sont granitiquas ct s'élèvent bien au-dessus de la ligne des forêts. Elles sont couvertes de ncige et de petits glaciers.

la riviène supérieure est un grand ruisseau glaciairc et beaucoup de petits cours d'eau se déversent dans le Jac. Celui-ci a 9 miles de long et environ i mile ou plus de large.

Depuis la base du lac jusqu'aux chutes de Stave, la rivière a 7 miles de long sur lesquels 2 miles environ sont pourvus de rapides d'une chute totale de I 1 pieds, le reste n'ayant pratiquement aucune chute.

Aux chutes mêmes et aux rapides, dans l'environnement immédiat des chutes de Stave, la rivière saute de 80 pieds $c t$ alors continue sa course par une série de rapides sur- une distance de 4 miles pour déboucher finalement dans un bassin où elle se joint avec la rivière Frather.

La chute totale depuis le niveau en basses caux du lac jusqu'à ce bassin est de 225 pieds. Et lorsque la digue aura ćté élevéc à sa hauteur définitive, l'ean sera surélevée de 35 pieds au-dessus du niveau des basses eaux du lac.

La digue formera un lac s'étendant des chutes de Stave jusqu’à la fin supéricure du lac de Stave sur unc distance de 16 miles. L'aire du lac avec la hauteur actuclle de la digue est environ 13 milles carrés et, lorsque la digue aura sa hauteur totale, elle atteindra 23 milles carrés. Lc réservoir aura une capacité de rí milliards de pieds cubiques.

L'étiage journalier du flot pendant 6 ans et surtout dans les trois dernières années a montré qu'un flot moyen de 3500 pieds cubiques par seconde pouvait être utilisé pour engendrer la force.

La chute totale depuis le lac jusqu'au bassin inférieur sera utilisée en 2 stations. La station supérieure qui actuel loment fonctionne, utilise r 25 pieds au maximum et 105 au minimum, et la station inférieure utilise la différence.
La place pour le premier développement fut choisie dans le voisinage des chutes du Stave, car, en ce point, la rivière cst divisée en deux branches par une petite île rocheuse d'un demi-mile de long. Cette situation est admirablement adaplée pour la construction d'une digue en amont et de travaux enfouis, tandis qu'un ancien chenal, le Blind-Slough, avec un lit rocheux de quelque six cents pieds de large, situé à ún niveau supérieur de celui de la rivière existante, présente la place la meilleure pour une digue permettant la décharge du flot.

Le second développement du projet consiste en une diguc de 160 pieds de haut à son point le plus élcvé, construite dans une gorge étroite pour former un réscrvoir de 3 miles de long, retenant l'eau jusqu'au niveau supérieur du canal creusé dans lo fond de la rivière.

La station de force sera placée à lest de la digue. Ic canal est creusé dans le fond de la rivière, au niveau de l'cau, en vue de la ligne principale du Canadian Pacific Railway. I.es canalisations pour les turbines seront placées dans des tunnels d'environ $\mathbf{. 6 0 0}$ pieds de long.

\section{DESCRIPTIOY DE TA STATION DE PUISSANCF}

La station de puissance telle qu'elle est actuellement consiste en une digue à écluses de 40 pieds de haut, munie de 5 écluses chacune de 22 pieds de large ; une digue solide de béton enfouie dans le sol ; 2 canalisations d'acier de $\mathrm{r} / \mathrm{f}$ pieds ayec de la place pour deux autres ; la maison de force avec 2 unités de ro ooo $\mathrm{Kw}$. et l'espace pour une extension deux fois plus grande; enfin un canal de 1500 pieds de long creusé dans l'ancien lit de la rivière.

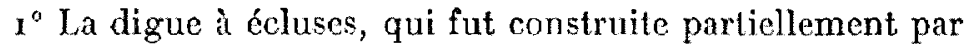
Fancienne Compagnie du lac Stave, consiste en quatre piles de 8 pieds de large avec 2 piles de soutenement, l'espace entre les piles étant rempli par des poutres de 24 inches et de 24 pieds de long qui peuvent être enlevées au moyen d'un treuil électrique permettant le passage de l'eau.

$2^{\circ}$ Digue enfouie dans le sol : les travaux cnfouis consistent en une digue solide de $7^{\circ}$ pieds de haut à son plus bas point, dans lesquels sont inclus les quatre canalisations qui ont 19 pieds de diamètre à l'ouverture et 14 pieds 6 pouces a l'endroit où elles quittent la digue. L'entrée de ces tuyaux de canalisation est ferméc par des portes d'acier radiales fermant une ouverture d'environ 20 pieds carrés. Ces portes sont mues par des moteurs électriques placés sur le sommet de la diguc. Il y a aussi 2 canalisations d'cxcitation avec les mêmes portes.

En avant des portes d'acier sont placés des écrans de très grande surface. Aucune précaution spéciale n'a été prise pour les protóger rle la glace, car la rivière Stave ne gèle pas. Ces digues sont actuellement construites de facon à élever l'eau d'environ 8 picds au-dessus de la surface la plus basse des eaux du lac. Elles seront élevées cette année de 10 pieds et les années suivantes de 2.5 .

I'année prochaine, une digue sera construite à travers Je Blind Hough. Flle sera constituée par des jetées en béton de 3o pieds de haut, laissant entre elles $\bar{x} / 4$ chemins d'ecluse, chacun clos par des blocs d'arrêts, qui peuvent être déplacés par un treuil électrique.

La digue dn Blind Hough pourvoiera pour un maximum de décharge de roo ooo pieds cubiques par scconde.

Les fondations de la station de force ont été excavées dans le roc solide où on a enlevé 75 ooo yards cubes.

Le canal crousé dans le fond de la rivière a 70 pieds de large et marchera avec une profondeur de ro pieds quand les 4 unités de la station de puissance seront en opération. Pour 
le construire on a enlevé 75 ooo cubes yards de sable, d'argile et de gravier.

Les fondations de la station de puissance sont d'une construction solide de béton et la superstructure de la maison est une formation combinéc d'acier et de béton armé. La construction est de 100 pieds de large et de 90 pieds de long. La chambre des turbines et des générateurs à $70 \times 80$ pieds et il $\mathrm{y}$ a deux chambres latérales de 28 pieds de large sur go pieds de long pour contenir les transformateurs de hautes et de basses tensions ; le tableau de contrôle est sur une galerie derrière une petite barrière, à l'extrémité du bàtiment.

Dans la station de force sont installés à présent 2 générateurs de ro ooo Kwa., triphasé, 6o tours par seconde et 4. 100 volts, construits par la Compagnie électrirque générate canadienne, actionnés par 2 turbines de 13 ooo chevanx-vapenr du lype Francis, construites par la Compagnie Bischer-Wyss de Zurich (Suisse). La figure o représente une de ces turbines.

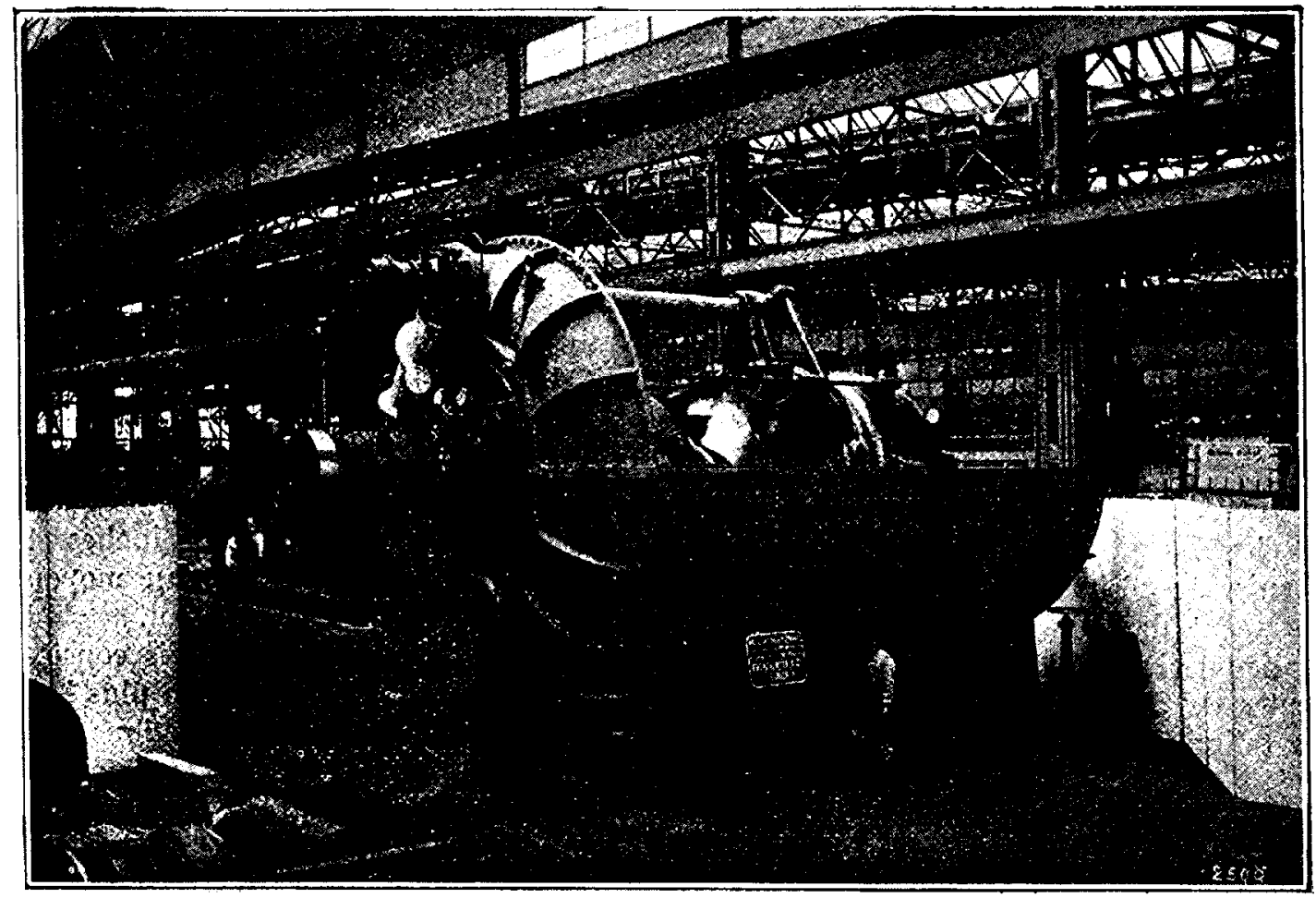

FIG. I. TURBINE ESCHER, WrSS ET C ${ }^{\circ}$, TYPE FRANGIS DOUBIE, DE STAVE I,AKE.

Puissance, 1300 II l' Haucur de chute, 36,3 metres ; debit, $38 \mathrm{~m}^{3}$; nombre de tours par minute, 225

L'excitation est fournic par 2 génératcurs de $250 \mathrm{Kw}$, I25 volts, chacun actionné par sa propre turbine et chacun capable d'exciter 4 machines. Six transformateurs monophasés de $3.000 \mathrm{Kw}$. de capacité sont situés sur le plancher principal, chacun dans sa propre voûte de béton avec une écoutille d'acier au sommet.

Le tableau de commande est dans une gralerie à l'extrémité Est du bâtiment, permettant ì l'opérateur d'avoir une vue complète de chaque pièce de la machincrie en mouvement.

Tous les transformateurs sont formés par des solénoïdes et avec les fusibles sont logés dans des petites cellules on béton renforcé dans unc rainure de ciment parallèle à la construction principale.

Il y a actuellement trois lignes de 60 ooo volts qui quittent la station, quatre de 13 ooo, et une de 4400 . Les circuits de 60000 volts s'en vont à travers des ouvertures dans le toit. Auparavant, les fils sont isolés dans le béton à partir de la rainure des fusibles. Des isolateurs sont montés directement au dessus de ces ouvertures. Les 13 ooo et 4000 volts sortent par le toit par des câbles triphasés sous plomb terminés en godets D. O. A. montés sur un support sur le toit.

L'installation est très satisfaisante, car le travail effectué pendant une annéc par un temps très mauvais n'a montré aucun défaut. La ligne principale de transmission de 32000 volts se termine à la station de réception à Ardley, point où le chemin de fer du Great Northern Railways est presque équidistant de Vancouver et New-Westminster.

L'installation de poteaux de transmission espacés de 660 pieds fut possible. Ils portaient deux circuits triphasés de càbles $n^{\circ}$ ro en bronze étiré dur avec un centre en étoupe et un fil de $3 / 8$ de inch en acier galvanisé, la hauteur du fil supérieur étant de 59 pieds et le point de support du fil le plus bas, fl pieds all-dessus de la terre. Des poteaux à ancres sont placés aux ungles aigus faits par la ligne ou aux points terminaux, capables de supporter deux fois l'effort maximum qui leur est demandé. La rivièro Pill, navigable, d'sl traversec sur une longueur de x 360 pieds ot il a été nécessaire de faire soulever la ligne à 100 pieds au point le plus bas. On a été obligé de recourir à une cons wuction spéciale : un poteau ell acier de 165 pieds (point supírieur, le fil le plus bas étant suspendu à ráo pieds du sol), fut élevé sur une pile de béton sur chaque bord de la rivière. Une tour spéciale est placée de telle sorte que les câbles à la traversée de la rivière (qui sont d'un $1 / 2$ inch de diamètre, en acier) font un angle de $45^{\circ}$ avee la ligne principale. la portée finit comme point mor à ces tours spéciales de chaque colé, de sorte que la tour principale n'a à supporter aucun effort elle-même. Il était nécessaire de mettre 2 séries d'isolateurs à chaque support, pour les points d'arrivée et de départ de cette portée, parce que les efforts étaient réellement trop grands pour un seul jou d'isolateurs. Ces tours spéciales sont fixées solidement par les câbles d'acicr de I inch de diamètre à des blocs de béton servant d'ancre. Tous les isolateurs étaient en faïence, fournis par la maison Ohio Brass Company. Trois disques de suspension de ro inches ayant été employés dans la construction de la ligne droite et 4 disques pour les points où il y a effort. Ies poteaux furent construits par Riter Conley Company de Pittsburg.

La $3^{\circ}$ ligne de 60 ooo volts court au Sud, vers la frontière inlernationale où elle rencontre la ligne installée par la $\mathrm{Pu}$ get Sound Traction Iight and Power Company, dans Bellingham. La distance totale élant de 47 miles, celle-ci est construite sur des poteaux de bois espacés de 200 pieds environ, la portéc la plus longuc étant de 97 pieds, à la traverséc de la Fraser River. Les poteaux de support sont, à cet endroit, de 130 pieds de haut, les isolateurs type Pin sont employés, le conducteur étant un numéro r équivalent; en 
acier à àme d'aluminium. Depuis que cette ligne a été en service, elle a eu à supporter un cyclone de $7^{\circ}$ miles à l'heure avec une température approximative de 32 degrés Farenheit. Dans deux autres circonstances, les fils ont cù à subir un dépôt de givre et de neige qui a porté leur diamètre à 2 inches. Ils n'ont subi aucune avaric.

Treize mille volts sont fournis pour distribution locale par l'usine motrice à une distance d'environ 20 miles. Les industries auxquelles on fournit cette force sont des scieries, fabriques de briques, pompes de drainage, carrières, etc. de plus, l'éclairage et la force sont donnés dans les villages trayersés par les lignes. Aucune stalion intermédiaire n'existe sur ces lignes. Les transformateurs qui réduisent à 2300 volts sont du type fait pour l'extérieur; ils sont placés sur un support au boul d'un poteau, aussi près que possible du service. Dans un ou deux cas où le consommateur demande une consommation suffisante pour garder un électricien, des transformateurs refroidis à l'eau sont placés dans de pelits batiments en fer, sous la surveillance drecel électricicn. Celte façon de distribuer est plus satisfaisante, à cause de la nature du pays et du caractère des affaires, que le système employé généralement consistant à placer de grandes stalions intermédiaires à des intervalles fixes d qui radient avec ${ }_{2} 300$ à 4000 volts.

I.s orérateurs des stations inlermédiaires sont ainsi éliminés. Le surveillant de section sur laquelle sc trouve le transformateur cst chargé de le surveiller. L'emploi du cuivre est réduit au minimum alin d'éviter la perte de ce rive dans les transformateurs.

Six lignes de $x 3$ ooo volts quitlunl la slation principale à Ardlor, qualre allant sur Vancouver cl deux à New-Westminster où le valuge esl réduit par des petites slations à 2300 volts pour la consommation locale. La force est distribuéc par ces petites stations à 2300 volts; 3 conducteurs sous papier couvert' de plomb, armés d'acier, sont posés directernent dans une canalisation de 30 inches de profondeur, sans garniture, disposés de la même façon qu'une conduitc d'eau. Des boîtes à joints sont employées. Des boîtes à sections sonl installées à des intervalles les plus commodes, et rendent possible l'isolement de petites sections sans détruirc l'équilibre du câble principal.

Comme la Compagnie cherche, surtout au début, à fournir de la force et non de la lumière, très peu de câbles sccondaires sont nécessaires, la majorité des services étant à 2300 volts. Dans les endroits où les services secondaires sont demandés, on place des transformateurs sur des poteaux, soit dans les propriétés privées, soit sur les chemins, les câbles se terminant dans des godets renforcés.

Le service secondaire peut aussi être souterrain, sous la forme de / conducteurs réunis pour I I5-200 volts, ces câbles sont réunis à chaud. On a cu très peu d'ennuis avec les cîbles. Il y a actuellement en service 25 miles de câbles, 9o miles du circuit à 6o ooo volts (exception faite des lignes de
Whatcom (iounly qui augmentent de r25 miles), i38 miles de 13 ooo volts, 2,66 miles de 2300 rolts, avec 7658 poteaux. 11 dépend de ces circuits 1157 clients, qui demandent I 55 r compleur's, et 57 ' 1 transformateurs de distribution avec capacité de 7606 kilowatts, sans compler les transformateurs pour i3 ooo volts. La demande maximum de force électromotrice qui a été faite à l'usine est jusqu à présent de 16 ooo kilowatts.

L'installation qui vient d'ètre décrite fournit un nouvel exemple de l'emploi de turbines de très grande puissance sous basse chute. Ici, où la hauteur de chute est relativement grande (36 mètres), on a domé le choix au type à axe horizontal, qui parait en effet la solution la plus rationnelle étant donné l'élat actucl de la technique, et co n'est point par les dispositions adoplées dans la construction de l'usine hydraulique que celle installation doit arrêter notre attention, c'est la puissance unilaire des groupes généralcurs : 13.000 chevaux, qui constilue une particularito remarquable dans le cas de cette hauteur de chule, puis- 


\section{LA CORREGTION DES TORRENTS}

I. Mourin, Inspecteur des Eaux et Forêts, auteur de l'inléressant ouvrage "Les Torrents de la Savoie " - dont nous avons publié dans notre dernier numéro un extrait très documenté - s'est ocupé d'une question fort importante, celle de la correction des torrents. Il a exposé ses idées à ce sujet dans une conférence au Congrès international forestier. I a compétence de M. Motsin sur ce sujet, nous fait un devoir de publier, en enticr, cet exposé plein d'enseigrnements qui intéresse si directement tous nos lecteurs.

"Depuis quelques années, les travaux de correction des torrenls ont cu lc don d'exciter, en France surtout, d'âpres et sourenl d'injustes critiques qui ont abouti à un véritable réquisitoire soumis à la fin de rgra ì la Chambre des Députés. (Rapport sur le budget de rgr3, Ministère de l'Agriculture par M. Albert Métin.)

Les asserlions erronées ou hasardées, avancées à ce sujet, onl fail l'objet de restrictions sévères de la part des foresliers ctrangers (Joumal forestier Suisse, rgog) aussi bien que de reclifications méritées, tant dans les journaux spéciaux (Revue des Eanx et Forèts rgo6, p. 75., Professcur Thierry) quà la tribune du Parlement (Séance de la Chambre dis rg novembre ig 2 , matin). La campagne entreprise ne tendait pas à moins qu'à affirmer l'inutilité, bien mieux, ha aocuité des travaux de correction.

En France, la loi du. 4 avril 1882 , en restreignant les travaux de restauration à la plaic et la lèvre même du torrent, en empêchant dans les bassins de réception l'établissement de massifs forestiers capables d'atténuer le ruissellement,,$a$ fatalement amené parfois à employer contre les brusques afflux d'caux et les érosions qui en sont la conséquence, de plus nombrcux ouvrages de correction qu'il n'eût été nécessaire dans des conditions normales.

Aucune polémique n'aurait dû naître à ce sujet : il suffit de rappeler comment procède l'action torrentielle pour faire apparaître l'opporlunité et souvent même la nécessité de ces ouvrages.

Tout filet liquide ayant une vilesse de plus de 3 mètres ì la seconde affouille les roches les plus dures comme lès granites. Lorsque ce filet roule des sables silicicux, l'action ćrodante de l'eau s'en trouve singulièrement augmentée ; il est clair aussi que la morsure du courant est d'autant plus énergique que la roche est plus tendre, plus soluble, plus friable.

C'est également une banalité de dire que la vitesse d'écoulement est fonction de la pente du lit et du volume des eaux, que le lapis vógétal forestier ou horbacé scrt de cuirasse in sol, ralenlit le ruissellement et absorbe une notable rartie des caux atmosphériques.

Or, le torrent est un cours d'eau à fortes pentes, lancé sur un vcrsant dénudé, souvent constitué par des boucs glaciaires, des marnes liasiques, ete, qu'il ronge sans cesse, amenant à chacuue crue de nouveaux éboulements des berges, de plus énergiques approfondissements du thalweg Tous ces matériaux arrachés à la montagne viennent s'étaler dans la valléc en énormes cônes de déjections, envahissant villages ct cultures, interrompant les communications et provoquant souvent dans les rivières interceptécs de désastrenses débâcles. Parfois même on a à déplorer des pertes de vies humaines.

Pour prévenir ecs dégâts, il fandrait diminuer l'importance des crues, supprimer ou réduire le ruisscllement, fixer la terre sur les versants : c'est le grand rôle de la forêt dans lc drame qui éclate lors de chaque orage en montagne. Mais suivant l'altitude, l'exposition, le sol, il faut i5 ans, 20 ans ct plus encore parfois, pour constituer le fourré protecteur. En attendant ce moment, n'y aura-t-il plus de "sac d'eau " capable d'élargir la plaie béante des rives et d'emporter en une lave les berges et les plantations qui les recouvrent? Est-il donc si rare de voir les neiges hivernales amoncelées dans les cirques supérieurs, fondre brusquement sous le souffle brûlant du vent du Sud, du fochn, qu'accompagnent presque toujours de tièdes et diluviennes ondées?

Alors que de vieilles futaies ont été impuissantes à retenir un sol étreint par le réseau de leurs monstrueuses racines, comment de jeunes pins, épicéas ou mélèzes, au maigre chevelu, arriveraient-ils à ravir à la puissance des eaux les pierrailles ou les boues qui les supportent ?

Si l'on veut permettre à la végétation de s'installer sur des versants instables, il faut de toute nécessité combattre l'érosion. Il peut suffire d'installer en travers du lit, à son niveau, cn divers endroits, une chaîne de pierres qui arrête le surcrcusement. Ces "seuils " assurent aux berges qui ont pris leur talus la fixitć qui leur faisait défaut, c'est là une correction simple et peu dispendieuse qui peut suffire en beaucoup de cas.

S'agit-il, au contraire, d'un torrent violent qui s'enfonce à chaque crue très profondément, sapant des berges presque verticalcs dont l'effondrement peut avoir dans le versant ainsi privé de base les plus fâcheuses répercussions.

Il convient alors d'édifier un mur en pierre sèche, ou en maçonneric de mortier, ou mixtc avec le corps de l'ouvrage en pierre sèche et le couronnement en maçonnerie de mortier, faisant saillie au-dessus du lit. L'atterrissement qui se forme en amont va élever la ligne du thalweg, étayer les berges croulantes et constituer une plage à pente réduite où les eaux s'étaleront, perdront de leur vitesse, de leur force de propulsion. La cuvette ménagée au millieu du couronnement du "barrage " éloignera le courant du pied des rives qui prendront peu à peu leur pente naturelle.

On conçoit d'ailleurs qu'il puisse être établi une série de barrages rapprochés, en gradins, lorsqu'il s'agira de déterminer un atterrissement important et de racheter une forte différence de niveau pour soutenir de puissantes berges menacées.

Ou bien chaque ouvrage se trouve établi à l'extrémité même de l'atterrissement du barrage immédiatement en aval ou à une distance plus considérable encore.

C'est la suppression de l'affouillement par suite de la diminution de la vitesse, due tant à la réduction de la pente qu'à l'augmentation du périmètre mouillé.

Dans cette dernière hypothèse, suivant la pente du lit, la nature du terrain, il peut être ou non nécessaire de fixer le thalweg par la construction de seuils intermédiaires.

Mais il arrive parfois que la pente est trop forte pour qu'on puisse utilement établir des barrages ou des seuils, on bien que le terrain est tellement peu consistant (éboulis, sable, gypse) que le moindre filet d'eau y produit des érosions. C'est alors que l'on recourt aux canaux perreyés : on assure au torrent un lit fixe, résistant, imperméable dans toute la région dangereuse. Pourrait-on concevoir autre chose, aussi bien pour protéger le sol que pour prévenir des infiltrations dont les effets, parfois lents à se manifester, sont souvent des plus redoutables?

Tous les moyens qui précèdent s'appliquent lorsque l'atfouillement est le seul ennemi à combattre. Le problème devient infiniment plus ardu lorsqu'à l'érosion vient s'ajouter l'instabilité des versants provoquée par des causes autrés 
que le surcreusement du lit. C'est toujours l'eau en excès qui est l'agent du glissement d'une couche superficielle filtrante (souvent un placage glaciaire) sur un substratum argileux ou rocheux imperméable, d'un relief souvent différent de celui de la superficie et plus ou moins incliné.

Le moutonnement de la surface, des déchirures vives, de petiles mares sont caractéristiques des glissements qui peuvent atteindre jusqu'à mille hectares et plus encore. Qu'un ruisseau coule dans le thalweg vers lequel se précipitent les terres, il se transformera en un dangereux torrent et plus il emportera de matériaux, plus la vitesse de descente du versant ira en augmentant.

Il sera toujours utile d'assécher les mares du bassin de réception, de drainer les parties mouilleuses, de faciliter, en un mot, la rapide évacuation des eaux atmosphériques ou de fonte de neiges, de manière à créer une croûte super. ficielle solide, formant une cuirasse capable de maintenir les masses humides sous-jacentes. En certains cas, de tels Iravaux constitueront à eux seuls toute la correction.

Mais ordinairement il conviendra de les associer à des ouvrages d'autres genres.

Tantôt cette digue devra être combinée avec des seuils ment, le long du cours d'eau de base dont on évitera l'affouillement, suffira à étayer le terrain.

Tautôt cette digue devra être combinée avec des seuils ou des barrages.

On peut aussi, en relevant fortement le lit du torrent au moyen de barrages qui constituent autant d'étais, provoquer de puissants atterrissements servant de cale à la berge mouvante.

Mais tous ces procédés demeurent inefficaces lorsque le torrent, d'un grand débit, est sujet à des crues violentes, soudaines et considérables, que la hauteur et la pente des versants instables sont fortes et que leur mouvement est rapide. Qu'on éloigne alors, si l'on peut, le cours d'ea'u de la rive dangereuse pour une dérivation à cicl ouvert ou en souterrain dans la rive opposée. D'un seul coup, on aura supprimé ou réduit énormément le charriage du torrent, on aura donné à la montagne croulante une place où entasser ses débris et l'appui dont elle a besoin pour retrouver son équilibre et sa stabilité.

Cette solution radicale, qui peut être économique, assure au mieux la sécurité des régions inférieures, des voies de communication, des cultures et des agglomérations dont les populations, quoi qu'on en ait dit, ne se désintéressent pas de la correction des torrents qui les menacent.

Moins chargées de matériaux et, par suite, plus affouillantes, los eaux auront une tendance à remanier leur lit à l'aval de la dérivation : de là l'obligation, pour éviter des surcreusements, de fixer ce lit au moyen d'une série de seuils qui réduiront la pente et partant la vilesse des filets liquides.

Il peut arriver aussi que le torrent dérivé soit jeté dans le lit d'un ruisseau voisin : ici encore, il faudra prendre garde que ce lit d'emprunt ne soit pas érodé par suite de la surcharge qu'on va lui imposer. Des ouvrages de prolection, de défense de rives seront sams doute nécessaires.

Sur les cônes de déjections enfin, qui portent les plus riches cultures, les vergers, les vignes, aussi bien que les villages, il n'est pas moins important de prévenir la divagation des caux torrentielles. Autrefois, alors que l'on ne cherchait pas à combattre le mal à son origine, dans ses causes d'ailleurs ignorées, on se bornait à des travaux de protection locaux, le plus souvent des digues, ou des curages de lit. A la première lave, on voyait les digues cn- lisées, le chenal ouvert à grands frais entièrement comblé, bien heurcux encore quand les coulées boueuses ne s'étaient pas frayé un chemin au travers des maisons el des champs.

Ce qu'on ne pouvait tenter alors avec suceśs se réalise aujourd'hui après l'achèvement des havaux de correction dont le résultat est la suppression du charriage.

D'ordinaire, on fixe un lit aussi rectiligne que possible, en tenant compte des points de sujétion (ponts, hameaux, etc.). Pour empêcher le torrent de sortir, on l'enserre entre deux perrés latéraux parallèles réunis car une cuvetle maconnée continue, ou par des seuils plus ou moins distants, afin d'éviter le creusement du plafond du chenal. Le premier de ces procédés a toutcfois l'inconvénient d'amener une usure du pavage de fond d'autant plus rapide que li pente du cône de déjections qu'il épouse est plus considérable.

Grâce à de telles régularisations de lit, on peut rendre à la culture des surfaces parfois fort imposantes qui n'étaient. occupées que par des graviers ou de la lande ; cn outre, on garantit contre les crues dont seul le reboiscment du bassin de réception du torrent atténuera peu à peu la violence, les propriétés, les routes et les chemins de fer situés dans la vallée principale.

Une autre question se pose, qui a élé soulevée récemment en France : faut-il commencer les travaux de correction par la partie inféricure du torrent et aller en remontant ou bien adopter la méthode inverse?

Voici ce qu'enseignait M. le professeur Thierry à l'Ecole Nationale des Eaux et Forêts (1).

"Il y a lieu de se demander s'il faut construire les barrages en commençant par le bas ou par le haut du torrent. Chacun de ces systèmes peut se soutenir. On peul commencer par le bas quand on est seulement préocempé de garantir immédiatement les propriétés inférieures ; on ne construit alors un nouveau barrage que lorsque le précédent est complètement attcrri, et de cette manière, on retient dans la montagne la totalité ou, au moins, la plus grande partie des gros matériaux.

" Cerlains reboiseurs pensent, au contraire, qu'il faut d'abord attaquer le torrent à sa source et construire les premiers barrages dans les parties supérieures des gorges. En opérant ainsi, on arrête immédiatement les dévastations dans les parties où les pentes sont les plus forles ; on régularise dans une certaine mesure l'écoulement des eaux et les perturbations inférieures deviennent moins considérables. Il est probable, pour toutes ces raisons, que ce système est plus économique que le précédent, mais il présente sur ce dernier le désavantage de ne pas mettre promptemenl à l'abri les cultures, les habitalions, les roules, les voies fer. rées qui sont menacées par le torrent.

" 11 existe un troisième procédé qui scrt d'inlermédiairs entre les deux autres et qui consiste à diviser lo torrent en un cerlain nombre de tronçons séparés par des bandes de terrain inaffouillables (affleurements de roches, veines plus résistantes, portions de lit devenues permanentes, elc.). Ces premiers travaux apporteront unc amélioration considérable dans le régime du torrent. Les propriétés inférieures seront garanties promplement, comme dans le premier cas ; et si, pendant la construction de ces ouvrages, on a soin de pousser aclivement les travaux de reboisement on pourra peutêtre, comme dans le deuxième cas, faire une économie séricuse sur le restant des travaux de consolidation à exécuter."

(1) Restauration des montagnes, Correction des torrents, Reboisement (Paris, librairie polytechnique, Baudry of Cie 6diteurs, Chapitre X, p. 145. 
Ces considérations pratiques, la liberté du choix, ne trouvent pas grâce devant les partisans de la correction par le bas qui condamnent les autres systèmes au nom du principe de l'érosion régressive des cours d'eau à partir d'un niveau de base et citent, à l'appui de leurs dires, géologues et géographes.

Il ne s'agit pas de nier cette théorie qui s'appuic sur des remarques incontestables, mais bien de voir si elle s'applique bien dans la lutte contre l'érosion torrentielle et la formation des laves. En de telles matières, le fait prime tout et s'il est contraire à une théorie, c'est que la théorie ne peut s'étendre au cas observé.

L'érosion torrentielle est provoquée par l'écoulement d'une masse liquide considérable, d'un "sac d'eau " tombé brusquement dans le bassin de réception. On voit aussi en quelques moments tomber 5o; 6o litres d'eau par mètre carré et souvent plus encore. Celte masse s'écoule sur des pentes excessives, sa vitesse s'accroît ( $g$ Sin $\alpha$ ) uniformément et, quand elle a atteint une valeur suffisante pour attaquer le lit, l'érosion commence. Il ne faut pas oublier que les roches les plus dures sont affouillées dès que l'eau atteint une vitesse de 3 mètres par seconde. Les filets liquides arrachent des malériaux au thalweg, les poussent et bientôt l'abondance des particules solides est telle que ce n'est plus de l'eau, mais un magma où la proportion du liquide est des plus faibles $(\mathbf{r} / 5, \mathbf{r} / \mathbf{1 0})$ et qui avance par bonds successifs. Viendra-t-on soutenir que les lois de l'hydraulique régissent encore l'écoulement de la lave?

Alors que la lave emprunte tous ses matériaux aux régions supćricures, peut-on valablement prétendre qu'elle est due à l'érosion régressive du cours d'eau ?

Ecoutez ce que dit Surell $\left(^{(}\right)$à ce sujet :

"Quand une grande masse d'eau se concentre subitement dans le goulot d'un bassin de réception, lancée sur une pente très rapide et resscrréc dans une gorge profonde, cette masse ne s'écoule plus suivant les règles ordinaires de l'hydraulique. Elle monte de suite jusqu'à une très grande haúteur, roule sur elle-mème el descend ainsi la gorge avec une vitesse excessive, bicn supérieure à celle du torrent d'eau régulier qui s'écoule devant elle vers l'aval. Elle doit donc alleindre successivement tous les points de ce courant; elle l'assimile à sa propre masse ; elle le balaye et, lorsqu'clle débouche dans la vallée, elle arrive chargée de tout le volume d'eau répandu dans le lit du torrent, depuis sa naissance, jusqu'à sa sortie de la gorge."

Qu'ajouler à une telle citation?

Mais où l'érosion régressive se fait d'une façon nelte, c'est après l'arrêt de la lave. Les matériaux se sont déposés formant une courbe convexe vers le ciel; lorsque les eaux plus claires qui succèdent au phénomène arrivent au contact de cet amas mou, sans cohésion, on les voit se creuser un chenal de plus en plus profond et donner au profil en travers du dépòt la forme d'un $\mathrm{M}$.

C'est à ces faits bien connus de tous ceux qui ont pratiqué les Alpes, qu'on veut opposer la théorie du creusement par des eaux claires courantes! Alors pourquoi ne pas dire que les lois de l'écoulement des liquides s'appliqueront aux laves?

Oì donc est la faute de lechnique que commettent ceux qui, dans les parties supérieures du torrent, veulent diminuer la vitesse des caux, augmenter la résistance du lit afin de prévenir l'érosion au point où clle se manifeste?

Surell, auquel il faut encore revenir, disait déjà à ce sujet $\left({ }^{2}\right)$ : "Il reste à parler de l'ordre dans lequel il con-

(1) Surrell, Etude sur les torrents des Hautes-Alpes, Chap. IX, p. 46.

(2) Surell, loc. cit. Chap. XXXII, p. 206, viendra de pousser les travaux. Cet ordre, loin d'être arbitraire, est une des conditions principales du succès.

" J'ai déjà si souvent fait ressortir dans le cours de ce travail la nécessité d'attaquer les torrents dans leurs sources mêmes, qu'il est inutile d'y revenir encore. Ainsi c'est dans les parties les plus élevées que les travaux seraient d'abord entrepris : ils avanceraient de là vers les parties basses."

Il faut, enfin, ne pas perdre de vue le but que l'on se propose en entreprenant la correction d'un torrent qui est la reconstitution de la forêt et le maintien du sol sur les pentes. Tout ce qui pourra hàter la réalisation de cet objectif ne saurait être négligé.

De là l'obligation de reboiser toutes les parties stables du bassin de réception afin d'atténuer le ruissellement, celle d'entreprendre des ouvrages de correction dans les régions supérieures pour fixer le lit, les berges, empêcher que celles-ci ne s'éboulent et n'entraînent peu à peu vers les thalwegs les parties les plus roisines.

(à suivre)

\section{ÉSSAI DES MATÉRIAUX}

Dans les "Annales des Travaux publics de Belgique," M. Paul Crustophe, Ingénieur principal des Ponts et Chaussées, fait un rapport sur le $\mathrm{VI}^{\ominus}$ Congrès de l'Association Internationale pour l'essai des malériaux. De ce rapport nous extrayons quelques points intéressants pour nos lecteurs. D'autres questions très importantes, telles que celles des matériaux de la route y ont été traitées, elles nous intéressent moins directement.

L'Association internationale pour l'essai des matériaux a tenu son sixième Congrès à New-York du 3 au 7 septembre rg12. Vingt Elats y avaient envoyé des délégués officiels. Sur soo congressistes, 250 étaient étrangers au nouveau continent.

Suivant la coutume des Congrès, outre les deux séances plénières présidées par M. le professeur Henry M. Howe, les travaux ont comporté des discussions en séances de sections. Celles-ci s'occupaicnt respectivement $: A$. des métaux, $B$. des ciments, pierres, bétons et béton armé, $C$. des matériaux diver's, sous la présidence respective de MM. Robert W. Hunt, R.-W. Lesley et Mansfield Merriman.

La tâche du Congrès était ardue.

Les publications distribuées aux membres ne comprenaient pas moins de 153 rapports consacrés aux questions les phus diverses, classcées en 29 calégories ( $\left.{ }^{1}\right)$.

Cet éparpillement extrême des sujets d'étude soumis an Congrès ne pouvait manquer d'amener un peu de confusion dans les discussions, bien que certains d'entre eux aient retenu plus particulièrement l'attention des membres prisents. Aussi n'y a-t-il pas lieu de s'étonner que les conclasions soumises au vote et adoptées en séance plénière ne donnent qu'une idée imparfaite de l'activité de l'Association.

Il ne saurait d'ailleurs être question, dans une matière aussi vaste et aussi perfectible que l'essai des matériaux, d'aboulir à des conclusions formelles et définitives.

Ce que l'on appelle matériaux, même dans le seul dómaine de la construction, comprend la nature entière dans ce qu'elle peut of ririr d'utilisable pour l'ingénieur.

Etablir des méthodes d'essai, ce qui est le but de l'Association, c'est rechercher comment il faut soumettre ces mar

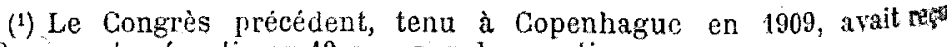
79 rapports répartis en 19 groupes de questions. 